\title{
Ultrafast 3-D Ultrasound Imaging using Row-Column Array specific Frame-Multiply-and-Sum Beamforming
}

\author{
Joseph Hansen-Shearer, Marcelo Lerendegui, Matthieu Toulemonde, Meng-Xing Tang*
}

\begin{abstract}
Row-column arrays have been shown to be able to generate 3-D ultrafast ultrasound images with an order of magnitude less independent electronic channels than traditional 2-D matrix arrays. Unfortunately, row-column array images suffer from major imaging artefacts due to high side-lobes, particularly when operating at high frame rates. This paper proposes a rowcolumn specific beamforming technique, for orthogonal plane wave transmissions, that exploits the incoherent nature of certain row-column array artefacts. A series of volumetric images are produced using row or column transmissions of 3-D plane waves. The voxel-wise geometric mean of the beamformed volumetric images from each row and column pair is taken prior to compounding, which drastically reduces the incoherent imaging artefacts in the resulting image compared to traditional coherent compounding. The effectiveness of this technique was demonstrated in silico and in vitro, and the results show a significant reduction in side-lobe level with over $16 \mathrm{~dB}$ improvement in sidelobe to main-lobe energy ratio. Significantly improved contrast was demonstrated with contrast ratio increased by $\sim 10 \mathrm{~dB}$ and generalised contrast-to-noise ratio increased by $158 \%$ when using the proposed new method compared to existing delay and sum during in vitro studies. The new technique allowed for higher quality 3-D imaging whilst maintaining high frame rate potential.
\end{abstract}

Index Terms-3-D Ultrasound, high frame rate / ultrafast imaging, real-time, Row-Column, Beamforming, Coherence, Frame multiply and Sum

\section{INTRODUCTION}

3-D ultrasound imaging is an emerging field in ultrasound research. Currently different techniques are used to produce 3-D images of the body using ultrasound. Ideally a fully addressed 2-D matrix array probe would be used [1], [2]. Unfortunately, these probes require a prohibitively high number of electronic channels. If a matrix probe has $N$ elements in the elevational direction and $M$ elements in the lateral direction, then for the matrix probe to be fully addressed it will require $N \times M$ elements and thus $N \times M$ electronic channels. Handling this quantity of channels is not feasible and thus alternative imaging techniques are required [3]. Many different approaches to solve 3-D imaging have been proposed and are currently being investigated, including both deterministic and optimised sparsely addressed matrix probes [4]-[9], multiplex matrix array probes [10], [11] mechanically steered 1-D probes [12] and micro-beaming techniques [13]-[15]. Sparse arrays under-address dense arrays, to reduce the element count

We would like to acknowledge the funding from the EPSRC CDT in Smart Medical Imaging, the EPSRC project grant (EP/T008970/1) and the Department of Bioengineering at Imperial College London. Dr Chee Hau Leow is also thanked for his contribution to the GPU beamforming code. *Email: mengxing.tang@imperial.ac.uk

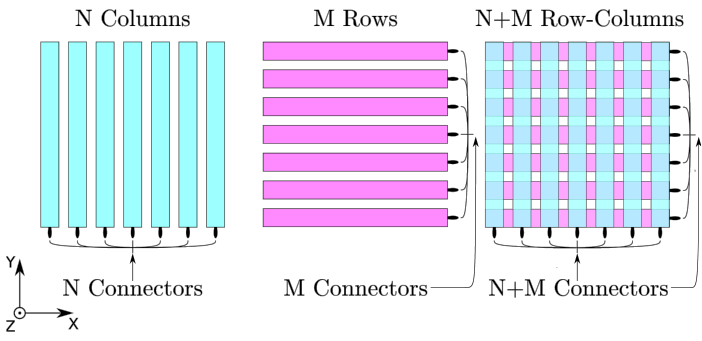

Figure 1: Diagram showing the basic configuration of the row-column array transducer. Where pink represents active area of row transmissions and turquoise represents column transmissions.

and hence the computational cost. However due to the large spacing between elements this imaging can lead to high grating lobes or high background artefact level and often a lack of uniformity in the pressure fields. Multiplexed arrays are full 2-D matrix probes that have many elements addressed to the same electrical channel. This leads to an increase in data acquisition time and a lack of flexibility. Mechanical steering involves either attaching a traditional 1-D array to a motor or localising a freehand scanner with an external sensor and acquiring a series of 2-D slices to form a 3-D image. These systems have a low frame rate, are limited by the positioning sensor accuracy, and in the freehand case can have gaps in volume where scan did not occur. Microbeamforming is a system whereby the transducer head includes application-specific integrated circuits, which implement the first steps in the beamforming algorithm. This technique is complex, expensive, and lacks flexibility. These methods have been reviewed recently [3]. This paper will focus on a different technique referred to as Row-Column Array (RCA) imaging [16].

An RCA probe consists of a set of elements aligned in one dimension (the rows) and another set of elements aligned orthogonally in a second dimension (the columns). All the elements are elongated in one dimension such that their footprint covers the entire probe surface, see Figure 1. By using an RCA probe the number of channels can be reduced to $N+M$. This greatly decreases the hardware requirement and computational cost of 3-D imaging. The RCA can either transmit using the rows or the columns. In reception again either the rows or the columns can be used. The transmitted beamforms are either a full plane wave using all elements in transmission, whereby the entire volume is imaged during each transmission, [17] or via cylindrical waves produced using a single element [18]. In the latter each of the elements is fired sequentially leading to a reduced frame rate compared to the full plane wave approach albeit with superior contrast. As this study is concentrating on fast 3-D ultrasound imaging only, 
the plane wave reconstruction technique will be considered. Since RCA probes were first introduced much work has been done to demonstrate the benefits of RCA probes [19]-[23], along with development of apodisation schemes to account for ghost echos and other artefacts [18], [24], high frame rate RCA imaging for flow estimation [25]-[28] and recently super resolution has been demonstrated using RCA probes [29].

Although RCA imaging is a promising technique for producing volumetric ultrasound images it suffers from imaging artefacts. When performing orthogonal plane wave compounding with Delay and Sum (DAS), artefacts located in the lateral and elevation direction, are large and 'cross-like' because each transmission event only has spatial information in two of the three dimensions. This means that the Point Spread Function (PSF) produced from a single transmission-reception event will have a line shape rather than a circular shape (as is the case with traditional ultrasound). Thus, to get complete information, an orthogonal transmission is needed for full spatial localisation. This will create a 'cross-shaped' PSF. Coherent compounding, using multiple transmission angles, can reduce the 'cross-shape' artefacts, but these are still prevalent particularly when fewer transmission angles are used. This is an issue during fast ultrasound imaging as fewer transmissions are used. The RCA probe also suffers from contrast issues due to a lack of elevational focusing typically present when performing 2-D imaging, these artefacts further reduce the contrast possible when using an RCA probe. For these reasons techniques which improve the contrast of images are needed for it to be able to compete with other 3-D imaging modalities.

Coherence beamforming is an emerging technique that would be used as an alternative for DAS beamforming with many different techniques having been recently proposed including Delay Multiply and Sum (DMAS) [30], Frame Multiply and Sum (FMAS) [31], coherence factor [32], [33], shortlag spatial coherence [34] and acoustic subaperture processing (ASAP) [35] amongst others. Compared to coherence-based beamforming using individual transducer element/data channel, such as DMAS and short-lag spatial coherence, coherencebased beamforming using imaging frames, such as FMAS can better take advantage of the highly complementary image frames generated by rows and columns in an RCA probe. The RCA probe is fundamentally spatially incoherent in the way it generates image artefacts as the column and row transmissions generate artefacts that are orthogonal to one another. In this context Bouzari et al. [36] has demonstrated improved focused imaging through extracting coherent information between row images and column images.

This study aims to exploit this incoherence by applying FMAS to a series of volumes produced via orthogonal plane wave imaging with multiple steering angles. Additionally, a new Row Column specific Frame Multiply and Sum (RCFMAS) algorithm was developed. These two methods will be evaluated in silico and in vitro and compared to traditional DAS beamforming. An optimisation sweep will be performed to evaluate the regimes where these new techniques are most effective. The new RC-FMAS method we propose aims to generate high contrast, low artefact ultrafast 3-D ultrasound using a coherence beamforming technique tailored specifically for RCA probes, which takes advantage of the incoherence of the row and column transmissions.

\section{MethodS}

\section{A. Beamforming}

1) Row-Column Delay and Sum: Prior to beamforming the data, In-phase Quadrature (IQ) Demodulation was applied to the raw data. This was done as the multiplication step of the FMAS and RC-FMAS step doubles any frequency component of the RF signals, requiring a doubling of the spatial sampling. However, IQ Demodulation will shift the central frequency to zero, allowing for the use of lower spatial sampling frequencies. When generating images with an RCA, an amended DAS algorithm needs to be used to account for elongated elements [18]. In this work volumes will be generated using a scheme of plane wave imaging developed by M. Flesch et al. [26], whereby transmissions are either steered using rows or columns. If the rows drive the steering, then columns will be used in reception and vice versa. In this way a series of volumetric intensity images $I_{D A S}$ are generated. After this coherent compounding can be used to generate a final volume. Figure $2 \mathrm{a}$ shows the pipeline used to generate row-column delay and sum images. In FMAS and RC-FMAS a different procedure, outlined below, will be followed.

2) Frame Multiply and Sum: FMAS is an existing technique already applied to $1-\mathrm{D}$ probes [31]. In this study it will be applied in 3-D for the first time. When performing FMAS each volume $\left(I_{D A S}\right)$, i.e. a 3-D image reconstructed using a single plane wave transmission, is coupled with the other volumes using all possible unique pairings. The resulting number of pairs $N_{P}$ is thus given by

$$
N_{P}=\left(\begin{array}{c}
N_{T x} \\
2
\end{array}\right)=\frac{N_{T x}^{2}-N_{T x}}{2}
$$

where $N_{T x}$ here is the total number of plane wave transmissions, including both row and column based transmissions. The paired volumes are then combined by taking the 'signed' geometric mean of the paired voxels. These combined pairs are then summed to give a resulting volume. If the product of the volumetric images $i$ and $j$ is given by

$$
I_{i j}(\overrightarrow{\mathbf{r}})=I_{D A S}(\overrightarrow{\mathbf{r}}, i) I_{D A S}(\overrightarrow{\mathbf{r}}, j),
$$

where $\overrightarrow{\mathbf{r}}$ is the position of the voxel, then the resulting delayed multiplied and summed volume $I_{F M A S}$ will be

$$
I_{F M A S}(\overrightarrow{\mathbf{r}})=\sum_{i=1}^{N_{T x}-1} \sum_{j=i+1}^{N_{T x}} \operatorname{sign}\left(I_{i j}(\overrightarrow{\mathbf{r}})\right) \cdot \sqrt{\left|I_{i j}(\overrightarrow{\mathbf{r}})\right|} .
$$

Here, the complex sign is taken to retain the phase information of the signal and the geometric mean of the paired volumes is taken to ensure the process is linear in amplitude. The final volume will then be produced by taking the normalised absolute value of this sum and then log compressing. Figure $2 b$ shows a schematic representation of the FMAS pipeline. 
3) Row-Column specific Frame Multiply and Sum: The technique outlined above is the direct application of the FMAS to RCA. Here we have developed a new algorithm which proposes to perform the multiplication in a more tailored way to take advantage of the incoherent nature of row transmissions and column transmissions data. A single volume produced from a single row transmission event will lack spatial information in the orthogonal dimension to the transmission, effectively a single point target will be stretched into a line. Coherently compounding multiple frames will give the resulting PSF from a single target a 'cross-like' appearance. By performing FMAS, but only pairing rowbased transmissions with column-based transmission, and not column with column or row with row the 'cross-like' effect can be greatly reduced, whilst keeping the noise reduction and other artefact suppression associated with current FMAS beamforming. Using this new technique the new number of pairs $N_{P}^{\prime}$ is also reduced and becomes

$$
N_{P}^{\prime}=N_{R_{t x}} \cdot N_{C_{t x}}
$$

where $N_{R_{t x}}$ and $N_{C_{t x}}$ are the number of rows transmissions and columns transmissions respectively. It should be noted that new number of pairs $N_{P}^{\prime}$ is significantly less than that in the direct implementation of FMAS shown in Eqn. (1). The new volume $\left(V_{R C F M A S}\right)$ will now be

$$
I_{R C F M A S}(\overrightarrow{\mathbf{r}})=\sum_{i=1}^{N_{R_{T x}}} \sum_{j=1}^{N_{C_{T x}}} \operatorname{sign}\left(I_{i j}(\overrightarrow{\mathbf{r}})\right) \cdot \sqrt{\left|I_{i j}(\overrightarrow{\mathbf{r}})\right|},
$$

where $i$ now represents the row transmissions and $j$ represents the column transmissions. Figure $2 \mathrm{c}$ shows gives a schematic representation of the new RCA specific FMAS pipeline.

\section{B. Simulations}

Simulations were conducted using the Field-II simulation environment [37], [38]. The RCA probe was simulated by generating two overlapping transducers, one representing the columns, one representing the rows. Each transducer consisted of 128 elements and was placed orthogonally to the other. Each row and each column were considered as one long thin transmission and reception element. The simulation parameters can be found in Table I. These settings were chosen as they matched the transducer parameters of a transducer which will be used for the in vitro verification and further in vivo work, see probe patent for details [39]. For row transmissions an impulse was given to the row elements and zero voltage was applied to the column elements and then in reception only the column channels were utilised. For column transmissions the opposite was the case. The plane waves were produced by applying delays to either the rows or the columns thus allowing for steering either in the elevational or lateral direction, respectively. For beamforming, an in-house beamformer was developed in the CUDA GPU programming environment. Three types of simulations were performed during this study. The first was a single scatterer, the response of which was investigated to generate PSFs of the different techniques. The
Table I: Simulation Parameters

\begin{tabular}{lll}
\hline & Value & Units \\
\hline Elements & $128+128$ & - \\
Frequency & 4 & $\mathrm{MHz}$ \\
Bandwidth & 101 & $\%$ \\
Speed of Sound & 1540 & $\mathrm{~m} / \mathrm{s}$ \\
Pitch & 0.2 & $\mathrm{~mm}$ \\
Element Length & 25.6 & $\mathrm{~mm}$ \\
Sampling Frequency & 80 & $\mathrm{MHz}$ \\
Receive Apodisation & Tukey (0.5) & - \\
Transmit Apodisation & Tukey (0.5) & - \\
Emission pulse & 1 cycle - Hann-weighted & - \\
\hline
\end{tabular}

second investigated multiple single scatterers at the same depth. The third was a tissue mimicking simulation.

1) Optimisation: To evaluate the imaging regime where the three techniques, DAS, FMAS and RC-FMAS operated most effectively, a simulation-based optimisation sweep was performing using varying transmission parameters. A point target was imaged at various depths from $10-50 \mathrm{~mm}$ using different numbers of angled transmissions at varying angle ranges. The PSF of each distinct imaging event was then compared using the Full Width Half Maximum (FWHM), Side-to-Main Lobe Energy Ratio (SMER) and Peak Side-to-Main Lobe Ratio (PSMR) as imaging metrics. These metrics are outlined in Section II-D. The number of angled transmissions ranged from 6 to 20 , where 6 transmissions means 3 column transmissions and 3 row transmissions. The angle ranges investigated varied from $1^{\circ}$ to $30^{\circ}$, where angle range is defined as difference between maximum and minimum angle used, i.e., an angle range of $10^{\circ}$ means angles from $-5^{\circ}$ to $5^{\circ}$ were investigated. By investigating the parameters outlined, optimal transmission parameters could be determined. For all tests the number of transmission angles and the ranges were kept the same for the rows and the columns. All remaining simulations and in vitro experiments were performed using 10 transmission angles with an angle range of $12^{\circ}$. This choice will be explained further in Section III. To evaluate the Signal to Noise Ratio (SNR) of the three techniques, additive white Gaussian noise was added to the Radio Frequency (RF) signal generated from a simulated point target located at $50 \mathrm{~mm}$. The noise was added prior to beamforming with an inputted SNR of $20 \mathrm{~dB}$. After beamforming, the SNR was calculated by taking regions of pure noise and comparing them to the intensity of the single point target.

2) Tissue Mimicking Phantom Simulation: The phantom used consisted of a series of point scatterers dispersed at random within the simulation volume with a Gaussian distribution of amplitudes, centred at zero. The structure investigated was composed of background scatterers along with a series of empty anechoic tubes positioned diagonally across the field of view. Figure 3 shows the tissue phantom used. The edges of the phantom were also left empty to demonstrate the effectiveness of the three methods in determining edges of regions and the impact of RCA artefacts on the final image quality. To ensure fully developed speckle pattern, 11 


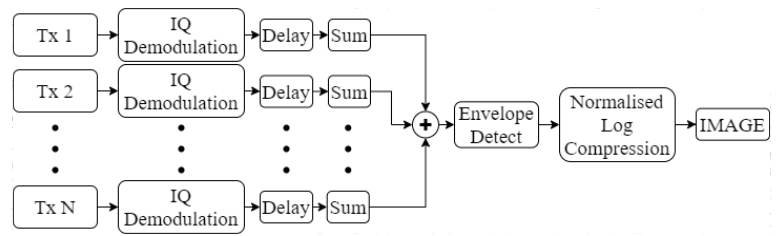

a Traditional Delay and Sum with Coherent Compounding Pipeline

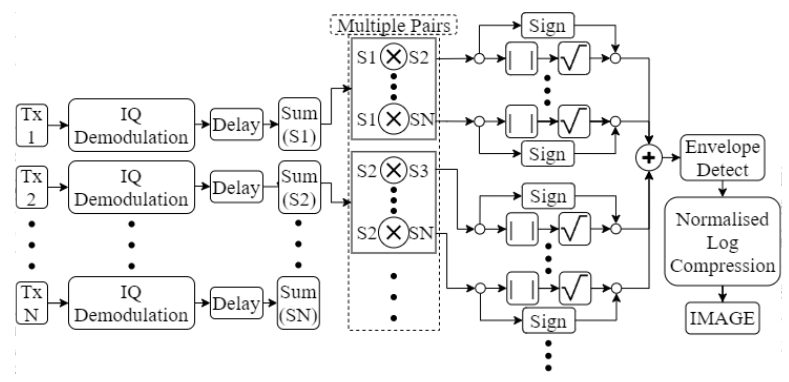

b Frame Multiply and Sum Pipeline

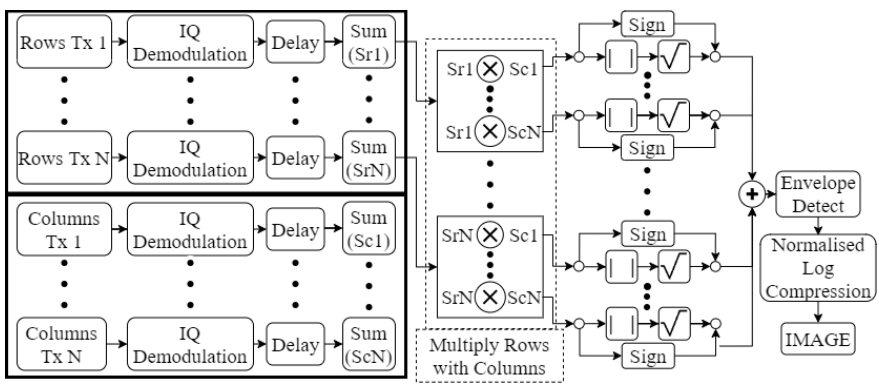

c Row-Column specific Frame Multiply and Sum Pipeline

Figure 2: Schematic pipelines for three different methods compared in this paper. Tx denotes a row transmission event which is then accompanied by a column reception or vice versa. In all schemes first 4 steps are producing images using traditional DAS method. After this the schemes diverge into three methods, simple coherent compounding, FMAS and RC-FMAS.

scatters were placed in each half wavelength cubic cell. White Gaussian noise was added before beamforming with a signal to noise ratio of $30 \mathrm{~dB}$ to the RF data to better replicate realistic ultrasound data. To compare the quality of the three imaging techniques with depth, two further imaging metrics were used: the Contrast Ratio (CR) and the generalised Contrast to Noise Ratio (gCNR).

\section{In Vitro Experiments}

Two in vitro demonstrations are also presented to further show the efficacy of the FMAS and RC-FMAS techniques. For in vitro experiments all imaging parameters are the same as outlined in the simulation section. For the experiments a Verasonics Vantage 256 platform (Verasonics Inc., Redmond, WA) was used along with an RCA probe [39] provided by Vermon S.A. (Tours, France). The first experiment was conducted using a stainless-steel wire of 50 microns in diameter. The wire was pulled taut and placed parallel to the probe face at a depth of $50 \mathrm{~mm}$. The second demonstration was a tube filled with contrast agent, which for this experiment was in-house prepared perfluorobutane microbubbles. Microbubbles were chosen as a convenient way to generate flow images with highly contrasting signal and non-signal regions to demonstrate RCA artefacts. They were prepared according to technique outline by C. Leow et al. [40]. For this

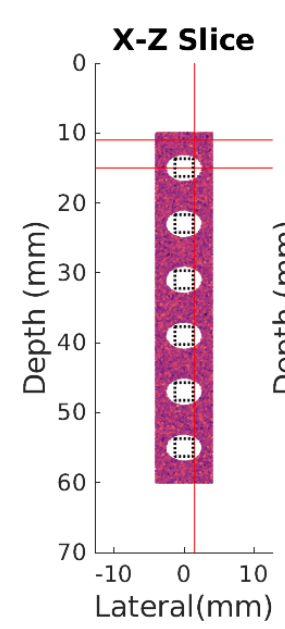

Field II Phantom Y-Z Slice

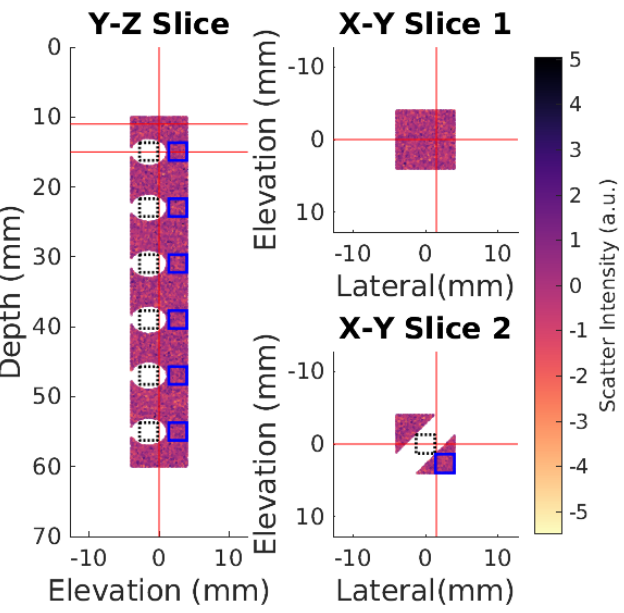

Figure 3: Cross sections of the Field-II phantom. The red lines represent the locations of the slices. These same slices are shown using the three methods, see Figure 8 . The black dotted and blue solid squares represent the cyst and tissue regions respectively used for calculating the $\mathrm{CR}$ and gCNR.

experiment the microbubbles were diluted such that they had a concentration of approximately $5 \times 10^{6}$ microbubbles $/ \mathrm{mL}$. The tube of inner diameter $6.5 \mathrm{~mm}$ was then imaged using a pulse inversion imaging sequence [41], whilst maintaining same imaging parameters as outline above. The tube containing the microbubbles was place at a depth of $30 \mathrm{~mm}$. A peristaltic pump with a flow rate of $200 \mathrm{ml} / \mathrm{min}$ was used to generate the flow. Using pulse inversion, with 10 transmission angles and imaging to a depth of $45 \mathrm{~mm}$ a frame rate of 770 frames per second is achievable.

\section{Image Quality Metrics}

The FWHM of the main-lobe of a single scatterer's PSF was used to give an indication of the resolution. The two metrics, SMER [42] and PSMR, were used to evaluate the image contrast. To quantify the image quality of the cyst phantom simulations and in-vitro tube experiments two further metrics were used: CR [43] and the gCNR [44].

The FWHM was calculated separately in all three dimensions. The elevational and lateral FWHM are equivalent due to the symmetry of the simulations. The SMER was calculated by taking the sum of the intensity of the side-lobes and dividing by the sum intensity of the main lobe. The main lobe cutoff point was set at $-6 \mathrm{~dB}$ and the side-lobes were bounded between $-40 \mathrm{~dB}$ and $-6 \mathrm{~dB}$. Thus the SMER is defined as

$$
\mathrm{SMER}=20 \log _{10}\left(\frac{\int_{-40 \mathrm{~dB}}^{-6 \mathrm{~dB}} \mathrm{I}(\overrightarrow{\mathbf{r}}) \mathrm{d} \overrightarrow{\mathbf{r}}}{\int_{-6 \mathrm{~dB}}^{0 \mathrm{~dB}} \mathrm{I}(\overrightarrow{\mathbf{r}}) \mathrm{d} \overrightarrow{\mathbf{r}}}\right),
$$

where $I(\overrightarrow{\mathbf{r}})$ is the intensity of voxel located at position $\overrightarrow{\mathbf{r}}$.

The PSMR was defined as the ratio of the amplitude of the maximum side peak divided by the amplitude of the main lobe. This metric will give an indication of the technique which produces the highest side-lobes whilst negating their extent. The CR is given by

$$
\mathrm{CR}=20 \log 10\left(\frac{\mu_{\text {tissue }}}{\mu_{\text {cyst }}}\right),
$$


where the $\mu_{\text {cyst }}$ is the mean intensity of region within cyst and $\mu_{\text {tissue }}$ is the mean intensity of tissue region at same depth, see Figure 3 for regions used. Intensities calculated on envelope detected data prior to log compression. The gCNR is given by

$$
\mathrm{gCNR}=\mathrm{C}_{0}{ }^{-\frac{\mathrm{C}_{0}}{\mathrm{C}_{0}-1}}-\mathrm{C}_{0}^{-\frac{1}{\mathrm{C}_{0}-1}} .
$$

where $C_{0}$ is the ratio of $\mu_{\text {cyst }}$ over $\mu_{\text {tissue }}$.

During the simulations, the SNR was calculated by taking the ratio of the intensity of the scatterer (signal) over the average intensity of a noise region. The noise region was chosen such that the entire region was absent of any scatterers and unaffected by the side-lobe of the point scatterer's PSF.

\section{RESUlts}

The first test performed was an optimisation sweep. The results, as seen in Figure 4, show the improvement to the image quality possible with both FMAS and RC-FMAS, with all imaging metrics improving. Choosing the optimum transmission scheme from these results is a non-trivial task as many factors need to be considered. For ultrafast imaging the frame rate needs to be as high as possible, thus a reduced number of transmissions is desirable. From Figure 4 it can be seen that the best contrast will be obtained with a large number of transmission angles as expected. The FWHM is largely independent of number of angles and relies much more heavily on the angle range. To optimise image quality all three metrics would ideally be minimised, whilst also taking into consideration desired frame rate for application in question. The primary goal of this new technique is to achieve reasonable imaging quality whilst operating in the high frame rate regime. For this reason, during the rest of the paper only the high frame rate regime will be investigated. For consistency all remaining experiments were conducted using the same imaging parameters. Ten transmissions angles (5 row transmissions and 5 column transmissions) and an angle range of $12^{\circ}$ was chosen. These parameters were chosen as provided good trade-off between all three imaging parameters whilst maintaining a high frame rate. Under these conditions the number of multiplicative pairs for FMAS and RC-FMAS will be 45 and 25 , respectively.

Table II summarises the results of the optimisation sweep for the imaging parameter chosen; errors shown are the standard deviation. From this, RC-FMAS is shown to outperform DAS in all metrics and outperforms FMAS in SMER and PSMR, with similar performance in remaining metrics.

Table II: Image quality metrics for point scatterer using 10 transmissions and $12^{\circ}$ angle range. Values shown are generated by placing scatterer at varying depths from 20 to $70 \mathrm{~mm}$ with an interval of $1 \mathrm{~mm}$. Errors given are the standard deviation.

\begin{tabular}{llll}
\hline & DAS & FMAS & RC-FMAS \\
\hline FWHM [mm] & $1.8 \pm 0.2$ & $1.5 \pm 0.2$ & $1.6 \pm 0.2$ \\
SMER [dB] & $30.8 \pm 1.1$ & $19.17 \pm 1.1$ & $14.8 \pm 1.0$ \\
PSMR [dB] & $-14.6 \pm 0.4$ & $-24.8 \pm 1.4$ & $-37 \pm 4$ \\
SNR [dB] & $51.2 \pm 1.5$ & $60.1 \pm 1.5$ & $57.0 \pm 01.5$ \\
\hline
\end{tabular}

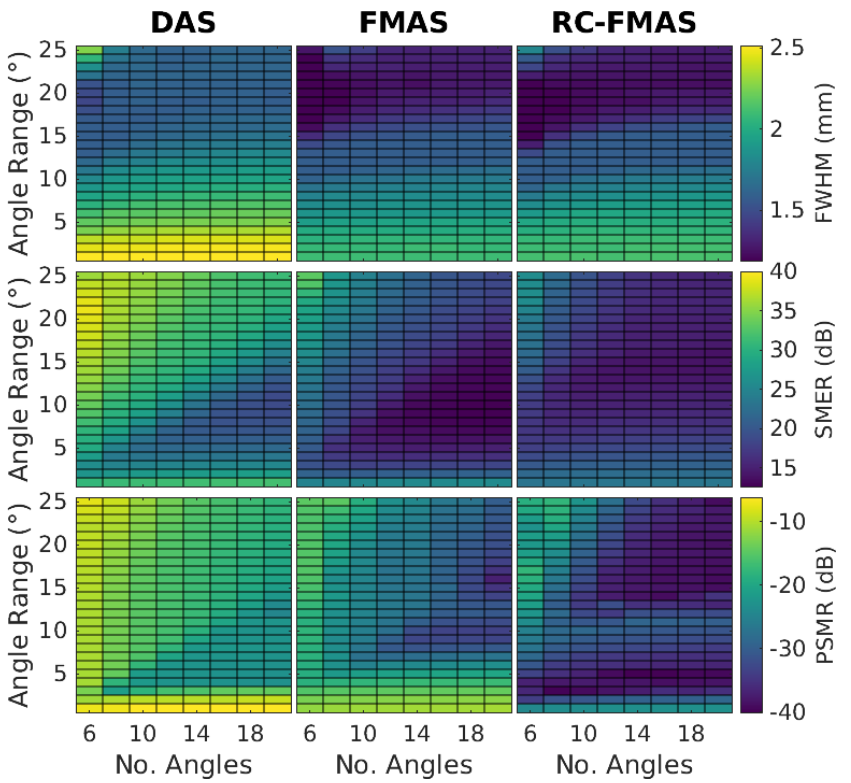

Figure 4: Results from the optimisation sweeps performed. The top row shows the FWHM of the central point source aligned with the $\mathrm{x}$ direction. The middle row shows the SMER, and the bottom row shows the PSMR. The FWHM gives indication of the resolution and the SMER and PSMR give indication of sidelobe reduction. The first column shows results from DAS beamforming, the middle column shows FMAS beamforming, and the final column shows RC-FMAS. In all plots the more purple the result the higher the image quality due to the various metrics. An angle range of $10^{\circ}$ means transmission angles from $-5^{\circ}$ to $5^{\circ}$ was investigate. The number of angles represents sum of row and column transmissions so 10 angles means 5 column based transmissions and 5 row based transmissions were used.

The SNR for this situation is also included for comparison. Figures 5 and 6a show the PSFs of the point targets imaged under the conditions outlined above. These two figures demonstrate the significant reduction in side-lobe intensity offered by FMAS and RC-FMAS. Note that there exists an artefact below each PSF which is referred to as a ghost echo. This can be removed by applying integrated apodisation to the RCA, as demonstrated by Rasmussen et al. [18]. This was not done in simulations here as it is not implemented in vitro. These and all future images are self-normalised after beamforming.

Figure 7 is an example when multiple scatterers are on the same plane. It demonstrates that due to the side-lobes artefacts it becomes harder to distinguish the multiple scatters from the artefacts and pinpoint their true locations, for example when performing super resolution, when high side-lobes are present. These artefacts get reduced when imaging using FMAS and RC-FMAS techniques. It should be noted that some specific situations the RC-FMAS technique has very similar performance to FMAS. This occurs when the side-lobes of one scatterer align with orthogonal side-lobe of another scatterer. In this scenario the added benefit of the RC-FMAS no longer applies. Both FMAS and RC-FMAS methods still outperform DAS in this situation. An example of this can be found in Figure 7, the centre scatterer's and the corner scatterer's sidelobes have coincided leading to little cancellation here. Where the side-lobes do not align the RC-FMAS method leads to the greatest reduction in side-lobe level.

Figures 8 and $6 \mathrm{~b}$ show in silico cyst phantom results. These figures demonstrate the image quality improvement offered by the FMAS method, and the new RC-FMAS method. Contrast 


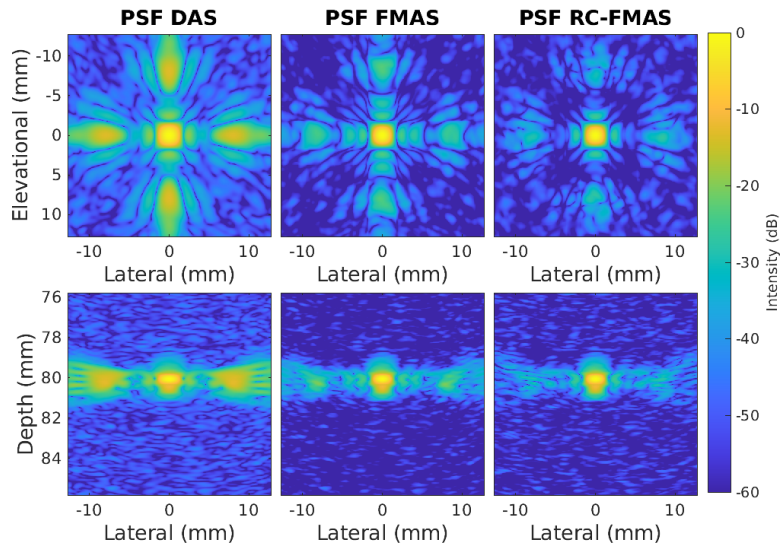

Figure 5: Example images of a point target. Plots compare the three methods (DAS, FMAS and RC-FMAS) with angle range of $12^{\circ}$ and 10 transmission angles being used. This is an example of the improvement possible when few transmission angles are used. Top row shows axial slice through the PSF, positioned at depth of $50 \mathrm{~mm}$ (location of point target). Bottom row shows lateral slice through the PSF, positioned at centre of transducer (location of point target).
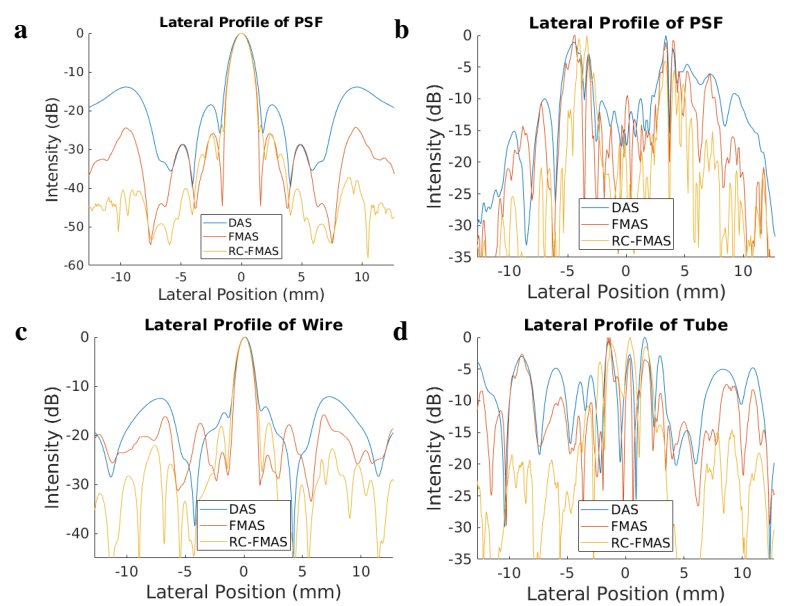

Figure 6: Lateral profiles of a) simulated PSF from point scatterer located at depth of $50 \mathrm{~mm} \mathrm{~b}$ ) simulated cyst phantom at $47.5 \mathrm{~mm} \mathrm{c}$ ) in vitro wire at $\sim 45 \mathrm{~mm}$ and d) in vitro microbubble filled tube at $\sim 30 \mathrm{~mm}$. Width of tube is $6.5 \mathrm{~mm}$. All results shown used an angle range of $12^{\circ}$ and 10 transmission angles.

is seen to be improved along with a reduction in the sidelobe artefacts located around higher scattering regions. Again, note that when side-lobes from rows and columns overlap the benefit from RC-FMAS is reduced, this can be seen at the ends of the tubes where side-lobes overlap the most. Overall, the new methods lead to 'sharper' delineation between cyst and surrounding phantom. The biggest improvement is seen at the sides of the phantom where 'mirror' images have been reduced significantly. The CR and the gCNR values were calculated for the three methods using the transmission scheme outlined above at different depths throughout the image, see Figure 9. While the image contrast measures vary over depth, RCFMAS always generates the highest image contrast measures, followed by FMAS.

Figures 10 and $6 c$ are examples of the in vitro results for the wire target using the 3 techniques. Significant sidelobe reduction is seen when using FMAS compared to DAS and a further slight reduction is seen when using new RCFMAS technique. Figure 11 is an in vitro demonstration,

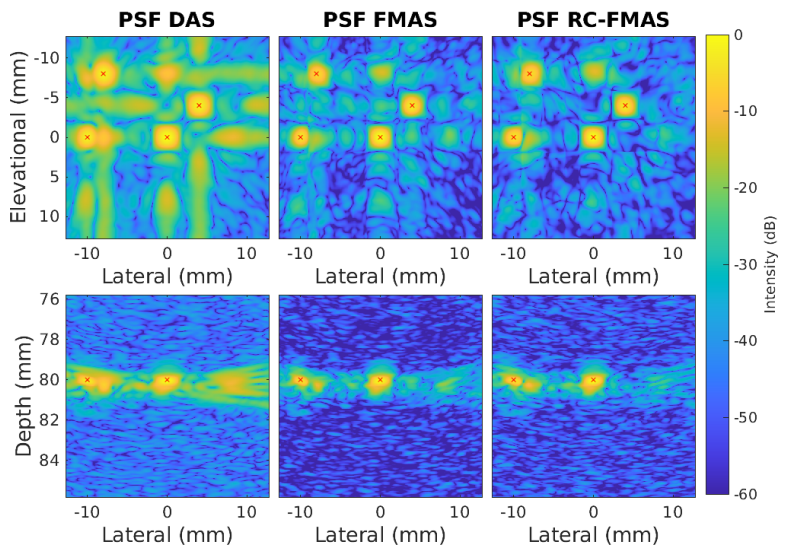

Figure 7: Simulation images of 4 point-scatterers located on the same plane reconstructed using the three beamforming methods. All imaging parameters are kept the same as Figure 5. The red crosses note the true location of the scatterers.

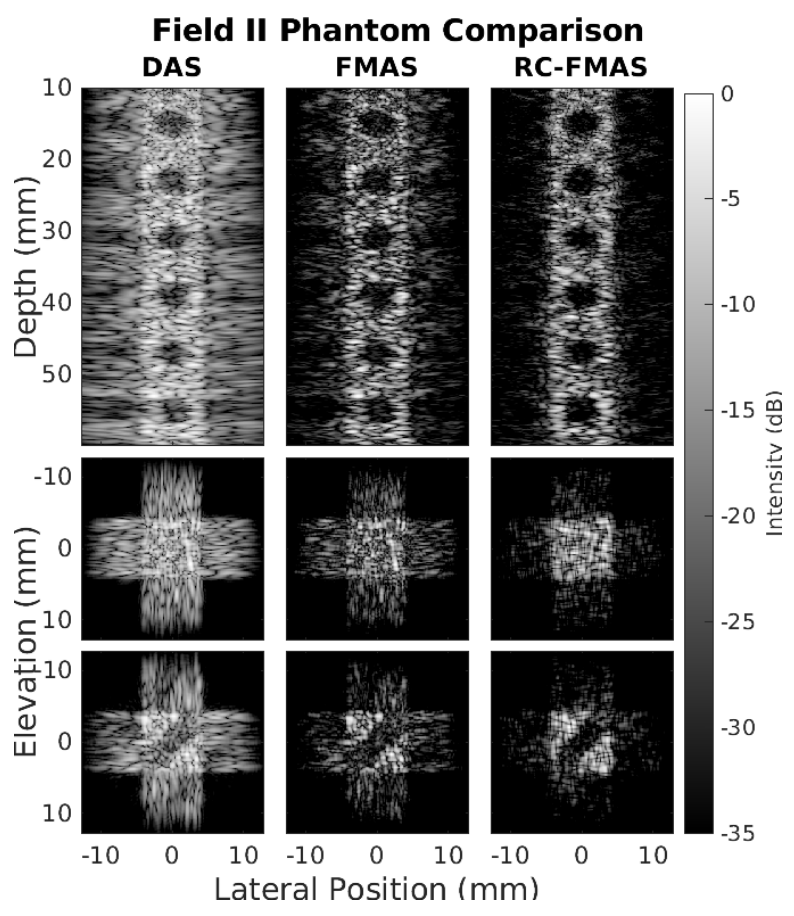

Figure 8: The cyst phantoms generated using Field-II. Left column is the standard DAS method, middle is the traditional FMAS method and the right column is the specific RC-FMAS method. Locations of the slices can be seen in Figure 3.

comparing the three techniques using the flow phantom and pulse inversion imaging. Again, the highest image quality can be seen in the RC-FMAS case. Videos of the flow comparing the three methods can be found in the supplementary material. The CR and gCNR was also calculated using the flow phantom in the same way as outlined during the simulation. The regions of interest used to generate these numbers were a cubic box wholly within the tube and a cubic box wholly outside the tube at same depth. The SNR was also calculated for the tube results with the signal region being taken within the tube and the noise region being region above tube that is filled with only water so can be assumed to just be noise signal. Multiple different regions were calculated and averaged to get a value and corresponding error. From Table III it can been seen that 

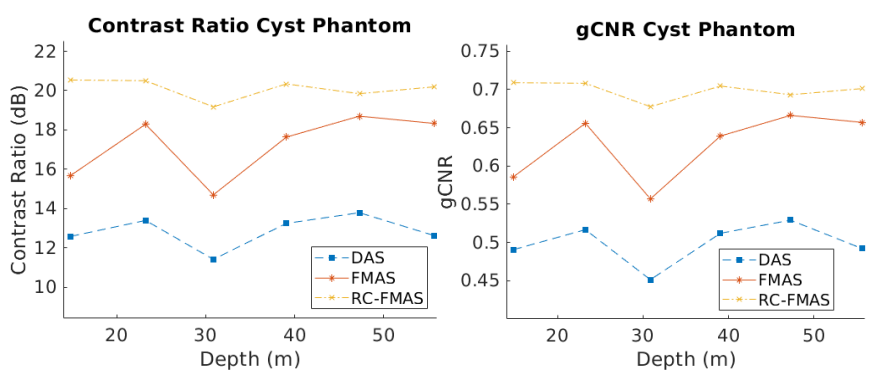

Figure 9: CR and gCNR values plotted at various depths. Regions used to calculate values shown can be seen in Figure 3.

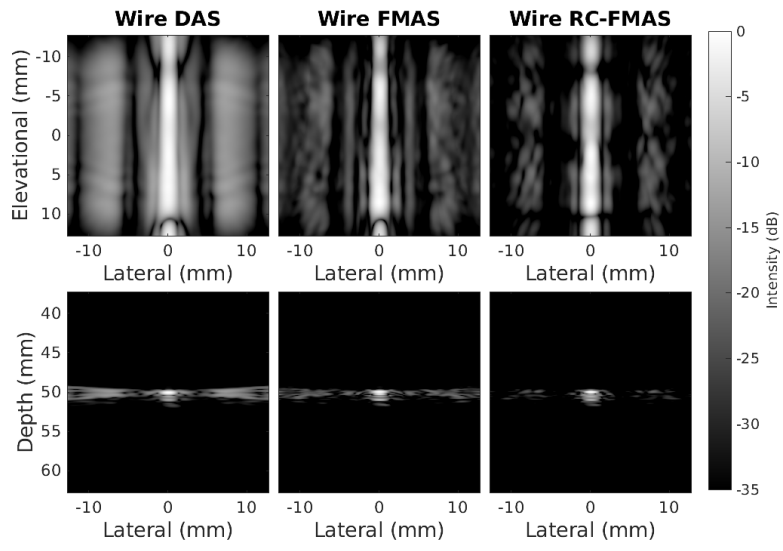

Figure 10: In vitro demonstration using the DAS, FMAS and RC-FMAS techniques with a stainless-steel wire as an imaging target. Top row shows axial slice of the wire located at $44.5 \mathrm{~mm}$. Bottom row shows lateral slice aligned with the centre of the probe.

RC-FMAS has improved all three image quality metrics with over $10 \mathrm{~dB}$ improvement in $\mathrm{CR}$, whilst the FMAS technique only led to an improvement of $1.4 \mathrm{~dB}$.

Table III: Image Quality Metrics for in vitro tube test.

\begin{tabular}{llll}
\hline & DAS & FMAS & RC-FMAS \\
\hline CR [dB] & $5.5 \pm 0.4$ & $6.9 \pm 0.5$ & $15.9 \pm 0.5$ \\
gCNR & $0.229 \pm 0.017$ & $0.286 \pm 0.019$ & $0.591 \pm 0.014$ \\
SNR [dB] & $29.0 \pm 0.5$ & $28.3 \pm 0.6$ & $31.7 \pm 0.8$ \\
\hline
\end{tabular}

\section{DISCUSSION}

By applying FMAS to theRCA probe, in silico artefact reduction was demonstrated during all tests, across all metrics. By amending the FMAS method, taking advantage of the intrinsic lack of coherence between row and column data, further improvement in SMER, PSMR, CR and gCNR was seen. In the high frame rate regime the SMER improved by $16 \mathrm{~dB}$, the PSMR by $23 \mathrm{~dB}$, the CR by $8 \mathrm{~dB}$ and the gCNR increased by $51 \%$. Using RC-FMAS the SNR and FWHM both improved compared to DAS and showed comparable performance to FMAS. During in vitro experiments similar improvements were also seen across the three techniques with RC-FMAS technique operating the best. In this case the CR improved by $10 \mathrm{~dB}$ and the gCNR increased by $158 \%$.

An optimisation sweep showed major artefact reduction during all acquisition schemes tested. From Figure 4 the general

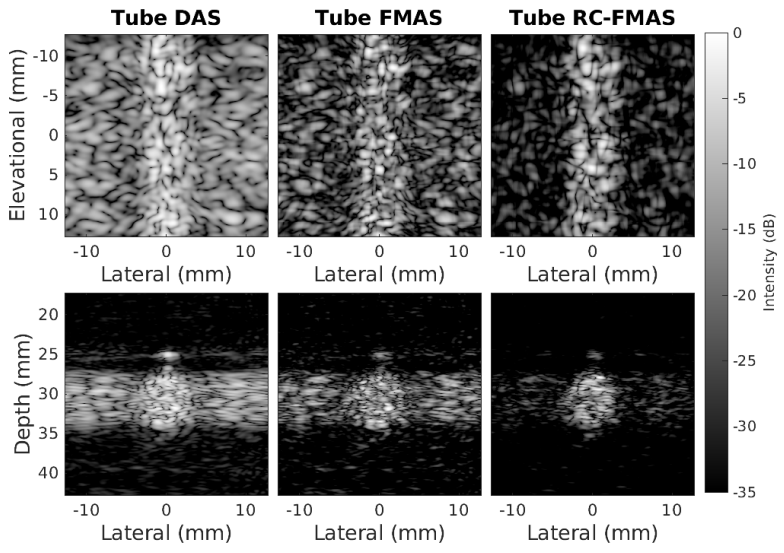

Figure 11: In vitro demonstration of technique in flow phantom consisting of a tube filled with in house produced microbubbles and imaged using pulse inversion. Top row shows axial slice of the phantom located at $30 \mathrm{~mm}$. Bottom row shows lateral slice aligned with the centre of the probe.

shape of the optimisation space did not change between the three methods, but improvement was seen when comparing DAS to FMAS and then again from FMAS to RC-FMAS, particularly when considering the SMER in a high frame rate setting. Both the FMAS and RC-FMAS methods lead to improvements in the FWHM of the point targets in lateral and elevational directions. The axial FWHM showed minor improvement with FMAS and RC-FMAS methods (results not shown as effect was minimal). The SMER and PSMR improved significantly using both new methods. As expected, the extremes (high angle range with low number of angles and low angle range) led to a reduction in PSF quality. When determining imaging parameters for future applications the interplay between number of transmission angles and angle range needs to be considered as different applications have different priorities. Additionally, the PSF quality will also be dependent on the depth. When imaging deeper points, angular range cannot be too high otherwise transmitted waves will no longer overlap and significant other artefacts will be introduced into the PSF. In this example the scatterers were imaged at multiple depths and the image quality metrics were then averaged whilst ensuring that all plane waves overlapped the target. When choosing appropriate imaging parameters for a given application, imaging depth of interest should be considered. These factors along with many others, such as frequency and pulse length need to be considered when designing imaging sequence. As this paper mainly focused on comparing the beamforming algorithms, these additional parameters were not studied. The most significant improvement was seen in the reduction of artefacts located horizontally away from the point target as shown in Figure 6a. The so-called cross-like artefacts can significantly reduce image quality. They produce mirror images of targets and thus will confuse diagnostic effectiveness of RCA imaging. For applications, such as super resolution, this can be a major problem as many false localisations may occur either side of a true localisation.

The results of the cyst phantom simulation and flow experiment, see Figures 8 and 11, demonstrates clearly that the contrast is greatly improved using both the FMAS method and RC-FMAS method. Quantitative improvement is also shown with significant improvement in both $\mathrm{CR}$ and $\mathrm{gCNR}$ when 
using the RC-FMAS versus any of the other two methods, as outlined above. This demonstrates that through RC-FMAS we can improve the reconstructed image quality. Through reducing image artefacts and improving the image quality, we expect that objects obscured by the noise and artefacts previously can be better visualised. An example of this is the increase in signal around higher scattering regions seen in DAS results shown in Figure 8. Without FMAS or RCFMAS this could be mistaken as a structure even though it is just a 'double' of nearby structure.

The in silico point target simulation found that the FMAS approach produced the best SNR. However, during in vitro tests the SNR for the RC-FMAS was best. It is suspected this might be due to some coherence in the noise generated by the rows/columns that is better eliminated when using RC-FMAS than when using FMAS. Alternatively, it could be caused by the flow, as the signal is not static there could be a reduction in the signal when more multiplications are performed as is the case with FMAS.

A major advantage of this method is that it allows for higher image quality compared to DAS when operating at high frame rates. Using fewer acquisition angles tends to reduce motion artefacts and aids in the imaging of fast-moving vessels, which is of particular importance when performing cardiac imaging. It will also facilitate faster acquisitions of super resolution images, which coupled with artefact reduction, should lead to better quality super resolution images with RCA probes.

It should be noted that FMAS and RC-FMAS is a slight increase in computational cost compared to coherent compounding. Equations 1 and 4 outlines the increase in computation using these new algorithms. With, the RC-FMAS technique having a reduced computational cost compared to the FMAS technique adding to the benefit of this new method. In the examples shown in this paper the number of multiplication pairs reduced from 45 when performing FMAS to 25 when performing RC-FMAS. It is also important to note that this increase in complexity is minimal compared to the complexity of the DAS algorithm which depends on the number of channels whereas the additional complexity of the new algorithms depends on the number of transmissions. As, RCA probes have a reduced number of channels compared to most other 3-D imaging techniques, the RC-FMAS technique uses a reduced number of multiplication pairs compared to traditional FMAS and the algorithms most expensive steps are fully parallelizable, the final computational cost will not be a significant challenge particularly with modern developments in Graphical Processing Units. For example, in the current implementation of the RC-FMAS technique, shown here the beamforming time increased by $\sim 0.015 \%$. It should also be noted that RCA imaging in general is the limited field of view. However different approaches, such as using a hardware diverging lens as proposed by $\mathrm{H}$. Bouzari et al. [45] solve this issue. As discussed earlier, when side-lobes overlap the benefits of FMAS and RC-FMAS is reduced, however there is less potential for overlap in the RC-FMAS method as only orthogonally aligned side-lobes will not cancel well. It should still be noted that the reduction in these situations is still greater than when performing just coherent compounding.
It does need to be acknowledged that the image quality is still not as high as current clinical 2-D imaging but by improving the image quality of RCA probes, via further optimisation and post processing techniques, RCAs should be to challenge any of the existing 3-D clinical systems due to their low cost and potential for higher quality images at high frame rates. Cardiovascular imaging, super resolution and cancer imaging/screening are all potential application areas of particular interest. To further prove the efficacy of this method, future in vivo demonstrations would be required

\section{CONCLUSION}

In this paper two methods for generating images using a row-column array and orthogonal plane wave transmissions were evaluated. The first FMAS method was an existing method which has been applied to the RCA probe for the first time. The second method was a new technique adapted from the FMAS specifically tailored for RCA probes. Across all metrics tested both the FMAS and RC-FMAS algorithms outperformed DAS and lead to significant image quality improvement. Further image improvement was then seen when using the RC-FMAS method compared with the FMAS method. The reduction in side-lobe artefacts has led to a clear improvement in B-mode images. In the future these methods will need to be tested in vivo. Additional post processing techniques and transmission parameter optimisations will need to be conducted before this technique can be applied clinically. They will then hopefully be able to improve on existing 3-D clinical technologies for ultrafast ultrasound imaging in a costeffective manner.

\section{REFERENCES}

[1] O. T. von Ramm and S. W. Smith, "Real time volumetric ultrasound imaging system," Journal of Digital Imaging, vol. 3, no. 4, pp. 261266, Nov. 1990.

[2] O. von Ramm, S. Smith, and H. Pavy, "High-speed ultrasound volumetric imaging system. II. parallel processing and image display," IEEE Transactions on Ultrasonics, Ferroelectrics, and Frequency Control, vol. 38, no. 2, pp. 109-115, Mar. 1991.

[3] Q. Huang and Z. Zeng, "A review on real-time 3d ultrasound imaging technology," BioMed Research International, vol. 2017, e6027029, Mar. 26, 2017.

[4] D. Turnbull and F. Foster, "Beam steering with pulsed twodimensional transducer arrays," IEEE Transactions on Ultrasonics, Ferroelectrics, and Frequency Control, vol. 38, no. 4, pp. 320-333, Jul. 1991

[5] A. Ramalli, E. Boni, A. S. Savoia, and P. Tortoli, "Density-tapered spiral arrays for ultrasound 3-d imaging," IEEE Transactions on Ultrasonics, Ferroelectrics, and Frequency Control, vol. 62, no. 8, pp. 1580-1588, Aug. 2015.

[6] R. E. Davidsen, J. A. Jensen, and S. W. Smith, "Two-dimensional random arrays for real time volumetric imaging," Ultrasonic Imaging, vol. 16, no. 3, pp. 143-163, Jul. 1, 1994.

[7] G. R. Lockwood, J. R. Talman, and S. S. Brunke, "Real-time 3-d ultrasound imaging using sparse synthetic aperture beamforming," IEEE Transactions on Ultrasonics, Ferroelectrics, and Frequency Control, vol. 45, no. 4, pp. 980-988, Jul. 1998.

[8] A. Austeng and S. Holm, "Sparse 2-d arrays for 3-d phased array imaging - design methods," IEEE Transactions on Ultrasonics, Ferroelectrics, and Frequency Control, vol. 49, no. 8, pp. 1073-1086, Aug. 2002.

[9] G. R. Lookwood and F. S. Foster, "Optimizing the radiation pattern of sparse periodic two-dimensional arrays," IEEE Transactions on Ultrasonics, Ferroelectrics, and Frequency Control, vol. 43, no. 1, pp. 15-19, Jan. 1996. 
[10] M. Bernal, B. Cunitz, D. Rohrbach, and R. Daigle, "High-framerate volume imaging using sparse-random-aperture compounding," Physics in Medicine \& Biology, vol. 65, no. 17, p. 175002 , Sep. 2020.

[11] J. Yu, H. Yoon, Y. M. Khalifa, and S. Y. Emelianov, "Design of a volumetric imaging sequence using a vantage-256 ultrasound research platform multiplexed with a 1024-element fully sampled matrix array," IEEE Transactions on Ultrasonics, Ferroelectrics, and Frequency Control, vol. 67, no. 2, pp. 248-257, Feb. 2020.

[12] A. Fenster, D. B. Downey, and H. N. Cardinal, "Three-dimensional ultrasound imaging," Physics in Medicine and Biology, vol. 46, no. 5, R67-R99, Apr. 2001.

[13] J. D. L. III, "2-d phased array ultrasound imaging system with distributed phasing," U.S. Patent 5229933A, Jul. 20, 1993.

[14] I. O. Wygant, N. S. Jamal, H. J. Lee, et al., "An integrated circuit with transmit beamforming flip-chip bonded to a 2-d CMUT array for 3-d ultrasound imaging," IEEE Transactions on Ultrasonics, Ferroelectrics, and Frequency Control, vol. 56, no. 10, pp. 2145-2156, Oct. 2009.

[15] G. Matrone, A. S. Savoia, M. Terenzi, G. Caliano, F. Quaglia, and G. Magenes, "A volumetric CMUT-based ultrasound imaging system simulator with integrated reception and $\mu$-beamforming electronics models," IEEE Transactions on Ultrasonics, Ferroelectrics, and Frequency Control, vol. 61, no. 5, pp. 792-804, May 2014.

[16] C. E. Morton and G. R. Lockwood, "Theoretical assessment of a crossed electrode 2-d array for 3-d imaging," in IEEE Symposium on Ultrasonics, 2003, vol. 1, Oct. 2003, 968-971 Vol.1.

[17] K. Chen, B. C. Lee, K. E. Thomenius, B. T. Khuri-Yakub, H.-S. Lee, and C. G. Sodini, "A column-row-parallel ultrasound imaging architecture for 3-d plane-wave imaging and tx second-order harmonic distortion reduction," IEEE Transactions on Ultrasonics, Ferroelectrics, and Frequency Control, vol. 65, no. 5, pp. 828-843, May 2018.

[18] M. F. Rasmussen, T. L. Christiansen, E. V. Thomsen, and J. A. Jensen, "3-d imaging using row-column-addressed arrays with integrated apodization - part i: Apodization design and line element beamforming," IEEE Transactions on Ultrasonics, Ferroelectrics, and Frequency Control, vol. 62, no. 5, pp. 947-958, May 2015.

[19] C. Seo and J. Yen, "5a-5 $64 \times 642$-d array transducer with rowcolumn addressing," 2006 IEEE Ultrasonics Symposium, 2006.

[20] A. Savoia, V. Bavaro, G. Caliano, et al., "P2b-4 crisscross 2d cMUT array: Beamforming strategy and synthetic $3 \mathrm{~d}$ imaging results," in 2007 IEEE Ultrasonics Symposium Proceedings, ISSN: 1051-0117, Oct. 2007, pp. 1514-1517.

[21] M. Pappalardo, G. Caliano, A. Caronti, et al., "Bidimensional ultrasonic array for volumetric imaging," pat. WO2008083876A3, Sep. 25, 2008.

[22] C. E. M. Demore, A. W. Joyce, K. Wall, and G. R. Lockwood, "Real-time volume imaging using a crossed electrode array," IEEE Transactions on Ultrasonics, Ferroelectrics, and Frequency Control, vol. 56, no. 6, pp. 1252-1261, Jun. 2009.

[23] A. Sampaleanu, P. Zhang, A. Kshirsagar, W. Moussa, and R. J. Zemp, "Top-orthogonal-to-bottom-electrode (TOBE) CMUT arrays for 3-d ultrasound imaging," IEEE transactions on ultrasonics, ferroelectrics, and frequency control, vol. 61, no. 2, pp. 266-276, Feb. 2014.

[24] T. L. Christiansen, M. F. Rasmussen, J. P. Bagge, L. N. Moesner, J. A. Jensen, and E. V. Thomsen, "3-d imaging using row-columnaddressed arrays with integrated apodization - part ii: Transducer fabrication and experimental results," IEEE Transactions on Ultrasonics, Ferroelectrics, and Frequency Control, vol. 62, no. 5, pp. 959-971, May 2015.

[25] S. Holbek, T. L. Christiansen, M. B. Stuart, C. Beers, E. V. Thomsen, and J. A. Jensen, "3-d vector flow estimation with row-columnaddressed arrays," IEEE transactions on ultrasonics, ferroelectrics, and frequency control, vol. 63, no. 11, pp. 1799-1814, 2016.

[26] M. Flesch, M. Pernot, J. Provost, et al., "4d in vivo ultrafast ultrasound imaging using a row-column addressed matrix and coherentlycompounded orthogonal plane waves," Physics in Medicine and Biology, vol. 62, no. 11, pp. 4571-4588, May 2017.

[27] J. Sauvage, M. Flesch, G. Férin, et al., "A large aperture row column addressed probe for in vivo $4 \mathrm{~d}$ ultrafast doppler ultrasound imaging," Physics in Medicine and Biology, vol. 63, no. 21, p. 215 012, 2018.

[28] M. Schou, L. T. Jørgensen, C. Beers, et al., "Fast 3-d velocity estimation in 4-d using a $62+62$ row-column addressed array," IEEE Transactions on Ultrasonics, Ferroelectrics, and Frequency Control, vol. 68, no. 3, pp. 608-623, Mar. 2021.
[29] J. A. Jensen, M. L. Ommen, S. H. Oygard, et al., "Three-dimensional super resolution imaging using a row-column array," IEEE transactions on ultrasonics, ferroelectrics, and frequency control, Oct. 21, 2019.

[30] G. Matrone, A. S. Savoia, G. Caliano, and G. Magenes, "The delay multiply and sum beamforming algorithm in ultrasound b-mode medical imaging," IEEE Transactions on Medical Imaging, vol. 34, no. 4, pp. 940-949, Apr. 2015.

[31] J. Kang, D. Go, I. Song, and Y. Yoo, "Ultrafast power doppler imaging using frame-multiply-and-sum-based nonlinear compounding," IEEE Transactions on Ultrasonics, Ferroelectrics, and Frequency Control, pp. 1-1, 2020.

[32] C. Yang, Y. Jiao, T. Jiang, Y. Xu, and Y. Cui, "A united sign coherence factor beamformer for coherent plane-wave compounding with improved contrast," Applied Sciences, vol. 10, no. 7, p. 2250, Jan. 2020.

[33] A. Fatemi, S.-E. Måsøy, and A. Rodriguez-Molares, "Row-columnbased coherence imaging using a 2-d array transducer: A row-based implementation," IEEE Transactions on Ultrasonics, Ferroelectrics, and Frequency Control, vol. 67, no. 11, pp. 2303-2311, Nov. 2020.

[34] M. A. Lediju, G. E. Trahey, B. C. Byram, and J. J. Dahl, "Short-lag spatial coherence of backscattered echoes: Imaging characteristics," IEEE transactions on ultrasonics, ferroelectrics, and frequency control, vol. 58, no. 7, pp. 1377-1388, Jul. 2011.

[35] A. Stanziola, C. H. Leow, E. Bazigou, P. D. Weinberg, and M.-X Tang, "ASAP: Super-contrast vasculature imaging using coherence analysis and high frame-rate contrast enhanced ultrasound," IEEE Transactions on Medical Imaging, vol. 37, no. 8, pp. 1847-1856, Aug. 2018.

[36] H. Bouzari, M. Engholm, M. B. Stuart, E. V. Thomsen, and J. A Jensen, "Improved focusing method for 3-d imaging using rowcolumn-addressed 2-d arrays," in 2017 IEEE International Ultrasonics Symposium (IUS), ISSN: 1948-5727, Sep. 2017, pp. 1-4.

[37] J. A. Jensen, "FIELD: A program for simulating ultrasound systems," in 10th Nordicbaltic Conference on Biomedical Imaging, Vol. 4, Supplement 1, Part 1:351-353, 1996, pp. 351-353.

[38] J. A. Jensen and N. B. Svendsen, "Calculation of pressure fields from arbitrarily shaped, apodized, and excited ultrasound transducers," IEEE Transactions on Ultrasonics, Ferroelectrics, and Frequency Control, vol. 39, no. 2, pp. 262-267, Mar. 1992.

[39] G. FERIN, M. Flesch, M.-C. Dumoux, D. Voisin, M. Legros, and A. Nguyen-Dinh, "Ultrasound transducer," U.S. Patent 20190328360A1, Oct. 31, 2019.

[40] C. H. Leow, E. Bazigou, R. J. Eckersley, A. C. H. Yu, P. D. Weinberg and M.X. Tang, "Flow velocity mapping using contrast enhanced high-frame-rate plane wave ultrasound and image tracking: Methods and initial in vitro and in vivo evaluation," Ultrasound in Medicine \& Biology, vol. 41, no. 11, pp. 2913-2925, Nov. 2015.

[41] D. Simpson, C. T. Chin, and P. Burns, "Pulse inversion doppler: A new method for detecting nonlinear echoes from microbubble contrast agents," IEEE Transactions on Ultrasonics, Ferroelectrics, and Frequency Control, vol. 46, no. 2, pp. 372-382, Mar. 1999.

[42] L. Tong, H. Gao, H. F. Choi, and J. D'hooge, "Comparison of conventional parallel beamforming with plane wave and diverging wave imaging for cardiac applications: A simulation study," IEEE Transactions on Ultrasonics, Ferroelectrics, and Frequency Control, vol. 59, no. 8, pp. 1654-1663, Aug. 2012.

[43] D. H. Turnbull, P. K. Lum, A. T. Kerr, and F. S. Foster, "Simulation of b-scan images from two-dimensional transducer arrays: Part i methods and quantitative contrast measurements," Ultrasonic Imaging, vol. 14, no. 4, pp. 323-343, Oct. 1, 1992.

[44] A. Rodriguez-Molares, O. M. H. Rindal, J. D'hooge, et al., "The generalized contrast-to-noise ratio: A formal definition for lesion detectability," IEEE Transactions on Ultrasonics, Ferroelectrics, and Frequency Control, vol. 67, no. 4, pp. 745-759, Apr. 2020.

[45] H. Bouzari, M. Engholm, C. Beers, et al., "Curvilinear 3-d imaging using row-column-addressed 2-d arrays with a diverging lens: Feasibility study," IEEE transactions on ultrasonics, ferroelectrics, and frequency control, vol. 64, no. 6, pp. 978-988, Jun. 2017. 